\title{
Some Effects of Viscosity on Wing-Body Interference at $M=1.9^{*}$
}

\author{
T. R. RHEES $\dagger$ AND W. P. CRENSHAW \\ University of Michigan
}

\begin{abstract}
Interference pressure distribution data were obtained at $M=1.9$ for the body of a flat rectangular wing-cylindrical body combination with a laminar boundary layer on the body. The results are compared with earlier experimental results obtained with a turbulent boundary layer on the body and with the linearized inviscid theory of NACA TN 2677.

The experimental results agree well with theory except for viscous effects not predicted by the theory.

Qualitative similarity of the shock-wave and laminar boundarylayer interaction to the two-dimensional case is noted.

Body lift loading due to interference for two configurations representing missiles with wings or control surfaces near the tail is discussed.
\end{abstract}

\section{Symbols}

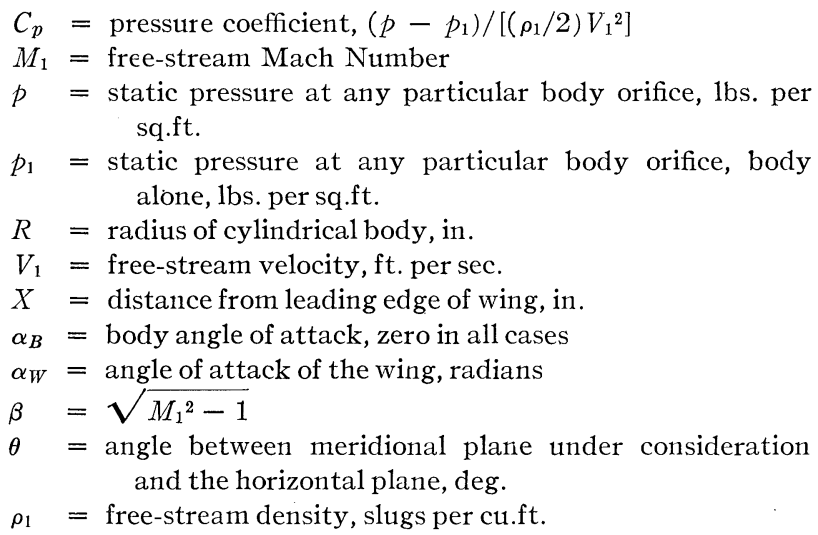

\section{(A) INTRODUCTION}

I $\mathrm{N}$ THE PAST FEW YEARS there has been considerable experimental and analytic study of the two-dimensional problem of the interaction of a shock wave and the boundary layer on a flat surface. In practice, however, the configurations in which shock waves interact with boundary layers are generally threedimensional. Wing-body combinations usually give rise to problems of the latter type.

Some of the methods that have been developed for computing the effects of wing-body interference on

Received December 6, 1954.

* This investigation was conducted at the University of Michigan Supersonic Wind Tunnel while the authors were continuing graduate study as part of the curriculum in Aeronautical Engineering of the U.S. Naval Postgraduate School, Monterey, Calif.

The authors are especially indebted to A. M. Kuethe, Felix Pawlowski Professor of Aerodynamics, and H. E. Bailey, Research Associate, Engineering Research Institute, for invaluable guidance and assistance.

$\dagger$ Lieutenant Commander, U.S. Navy.

$\ddagger$ Lieutenant, U.S. Navy. pressure distribution in a supersonic flow have been summarized and compared by Lawrence and Flax ${ }^{1}$ and Phinney. ${ }^{2} \quad$ A method of solving a wide class of wingbody interference problems is presented by Nielsen and Pitts, ${ }^{3}$ with a numerical application to the case of a flat rectangular wing mounted at an angle of attack on a cylindrical body at zero angle of attack. The extension of the method for the body at an angle of attack has been made by Bailey and Phinney ${ }^{4}$ and by Pitts, Nielsen, and Gionfriddo. ${ }^{5}$

The experimental interference pressure distribution on the body of a wing-body combination in a supersonic flow differs from the theoretical inviscid pressure distribution due to two effects: (1) the presence of subsonic velocities in the boundary layer permits upstream propagation of pressure disturbances within the subsonic portion of the boundary layer, thus influencing the flow field ahead of the Mach cone whose apex is at the disturbance; and (2) the sharp pressure gradients that may exist in a supersonic stream can induce sizable cross flows in the three-dimensional boundary layer and even separation of the boundary layer.

For the particular wing-body combination used in this investigation, Bailey and Phinney, ${ }^{6} 7$ have presented the theoretical inviscid interference pressure profiles on the body and experimental interference pressure profiles measured with a turbulent boundary layer on the body.

It was the purpose of this investigation to obtain the interference pressure profiles on the body with a laminar boundary layer on the fore body and to compare the results with the experimental results for the turbulent case $^{6,7}$ and the theoretical pressure distribution predicted by the method of Nielsen. ${ }^{3}$ In addition, it was desired to compare the predicted body lift due to interference for two possible wing-body configurations with the experimental force when the boundary layer on the body was laminar and when turbulent.

The Reynolds Number for these tests, based on a 2 -in. model body diameter, was $7 \times 10^{5}$, which corresponds to an actual missile of 2 -ft. diameter flying at Mach Number 1.9 at $90,000 \mathrm{ft}$.

\section{(B) Equipment and Procedure}

The experiments were conducted in the University of Michigan 8 by 13 in. Intermittent-Flow Supersonic Wind Tunnel, ${ }^{8}$ Mach 1.9 channel. 


\section{(1) Description of Model}

The model consists of a cylindrical body and two half-wings, Fig. 1. The model is the same used by Bailey and Phinney ${ }^{6} 7$ except for use of a shorter nose piece, ensuring a laminar boundary layer on the body in the test region. Use of the same model permits a direct quantitative comparison between the results obtained for the laminar case and the results reported for the turbulent case.

The model body is a hollow circular cylinder, with a 2 -in. outer diameter and a $1 \frac{1}{2} 2^{-i n}$. inner diameter. The nose piece is $2^{9} / 16$ in. long and tapers to an inlet diameter of $1^{5} / 16$ in. The chord of the half-wings is 2 in. and the half-span is $2^{1 / 2}$ in. The wing section is flat on one side and double-wedge shaped on the other, with a wedge angle of $10^{\circ}$ at the leading and trailing edges.

The body contains 92 pressure orifices arranged in 21 meridional planes, with 1 in. between the orifices in each meridional plane. In the present investigation, 44 of the pressure orifices in the upper quadrant were used.

The two half-wings are rigidly supported by brackets outside the test section. The sealing of the wing-body juncture was accomplished by mechanical contact at flat spots on the side of the body. The maximum gap encountered was slightly greater than 0.008 in. at the leading edge of the wing.

The axial position of the body may be varied about $1 \frac{1}{2}$ inches by axial movement of the strut attachment at the top of the tunnel. Since the half-wings are fixed with respect to the tunnel, this axial movement of the body permits the measurement of pressure profiles on the body in the presence of the wing. The axial position of the body and thus the orifice locations with respect to the leading edge of the wing was determined within \pm 0.003 in.

\section{(2) Instrumentation}

The static pressures were measured with mercury manometers which were photographed during the test run, permitting an accuracy in the measurements of 1 per cent of the ambient static pressure.

Schlieren photographs of each configuration were obtained.

A modified china-clay technique was used to illustrate the flow in the boundary layer close to the surface of the body. The drying time was controlled so that a dry film was obtained for the laminar boundary layer and a wet surface would aid in identifying a region of separation. The china-clay pattern was also useful in locating the traces of shock waves on the body and wing surfaces.

\section{(3) Experimental Procedure}

Prior to taking data, two runs were made with the body alone: (1) visual schlieren showed nose shock attached and no blocking; and (2) china-clay pattern indicated a laminar boundary layer over the fore part of the body.

For all runs, zero body angle of attack with respect to the free stream was maintained. Pressure data were obtained with the half-wings positioned at $\pm 8.2^{\circ}$ angle of attack. The variation of angle of attack setting between runs was negligible. Pressure data were also obtained for the body alone.

The china-clay pattern was checked on every run to ensure that the boundary layer ahead of the Mach helix originating at the juncture of the leading edge of the wing and the body remained laminar, and pressure readings in three meridional planes were plotted after each run to check immediately the reliability of the pressure data for that run.

\section{(4) Data Preparation}

The pressure measured at any one orifice with the body alone in the tunnel may be written

$$
p_{\text {measured }}=p_{\text {body alone }}+\Delta p_{\text {induced }}
$$

where $p_{\text {body alone }}$ is the pressure on the body alone in a uniform flow and is a function only of the geometry of the particular body and $\Delta p_{\text {induced }}$ is the incremental pressure induced on the body alone due to the windtunnel flow nonuniformities and is a function of the body geometry and the flow nonuniformities.

If the wing is introduced into the flow, the measured pressure becomes

$$
p_{\text {measured }}=p_{\text {body alone }}+\Delta p_{\text {induced }}^{\prime}+\Delta p_{\text {interference }}
$$

where $p_{\text {body alone }}$ is as defined above, $\Delta p^{\prime}$ induced is the incremental pressure induced on the body in the presence of the wing due to the wind-tunnel flow nonuniformities, and $\Delta p_{\text {interference }}$ is the incremental pressure induced on the body in a uniform flow due to the presence of the wing. Uniform flow is the condition usually encountered in free flight but is extremely difficult to obtain in a wind tunnel.

Provided $\Delta p_{\text {interference, }} \Delta p_{\text {induced }}$, and $\Delta p^{\prime}{ }_{\text {induced }}$ are small quantities,

$$
\Delta p_{\text {induced }}=\Delta p^{\prime}{ }_{\text {induced }}
$$

so that a simple subtraction of the pressure on the body alone from the pressure on the body in the presence

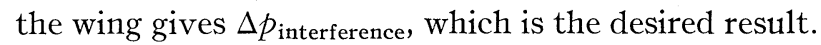

Actually, there is some interaction between $\Delta p_{\text {interference }}$ and $\Delta p_{\text {induced }}$, as evidenced by a small axial shift of a weak tunnel shock observed in the bodyalone pressure data when the wing is introduced into the flow. This shift was taken into account when fairing the raw experimental pressure curves.

The experimental interference pressure profiles were obtained by a simple subtraction of the faired pressure profiles for the body alone from the faired pressure profiles of the wing-body combination. In this manner, the effect of tunnel flow nonuniformities is eliminated as far as possible.

The zero base for all curves presented herein is the static pressure measured on the body alone. It should 
be noted that, although the curves are in effect nondimensionalized for presentation, they apply strictly only for the particular angle of attack used. It has been observed that the pressure distribution on the body, presented in terms of $\beta C_{p} / \alpha_{W}$, is affected by the angle of attack of the wing. ${ }^{5,} 6$

\section{(C) Results and Discussion}

From the schlieren photograph of the body alone, Fig. 2, the laminar boundary-layer thickness measured at a point 6 in. from the nose is between 0.025 and 0.030 in. Fig. 3 shows a somewhat thicker boundary layer at this position after the wing has been introduced. For comparison, the compressible laminar boundary-layer thickness for 6 in. of flat plate is determined theoretically to be 0.029 in. $^{9}$ In subsequent references to the upstream influence of the interference pressure in terms of boundary-layer thicknesses, the thickness will be assumed 0.03 in.

Experimental interference pressure profiles for four representative meridional planes and the corresponding theoretical profiles are plotted in Figs. 6a, 6b, 6c, and $6 \mathrm{~d}$. Inasmuch as the theory cannot be applied downstream of the trailing edge Mach helix of the wing, the theoretical pressure was assumed to decrease abruptly to ambient pressure along that helix. All interference pressures were measured on the quadrant of the body above the flat surface of the wing, with the wing fixed at $\pm 8.2^{\circ}$ angle of attack. Since the model is axially symmetric and the body was maintained at zero angle of attack, the pressure data measured for the two angles of attack of the wing in effect gave the pressure distribution on the body in the presence of a plate-plate wing.

In a comparison of the experimental results with theory, for a particular meridional plane of the body (Fig. 6), two factors affecting the axial position of the wing-body phenomena must be considered: (1) The theoretical pressure discontinuity occurs along the Mach wave helix originating at the linearized position of the wing-body leading- and trailing-edge juncturesi.e., in the plane $\theta=0^{\circ}$. Since the wing plane is actually inclined $8.2^{\circ}$, the helices are shifted axially from the linearized position by an amount $X / \beta R=0.15$. (2) Occurrence of the actual finite pressure jump along the trace of an inclined shock, rather than a Mach wave, introduces a wave-angle error.

\section{(1) Wing at $-8.2^{\circ}$ Angle of Attack}

For a negative wing angle of attack, a compression occurs over the flat surface of the wing. In an ideal fluid, the interference pressure coefficient on the body is zero ahead of the Mach helix generated at the leading-edge juncture; the pressure rise at the helix is a discontinuous shock jump, followed by a continuous axial pressure gradient due to the interference potential field. The magnitude of the shock jump at the leadingedge helix is continuously attenuated as the shock moves around the cylinder, diminishing to zero theoretically at the $\theta=90^{\circ}$ meridian arc.
Experimentally, with laminar boundary layer on the body, upstream propagation of the pressure disturbance varies from about 30 boundary-layer thicknesses in the $\theta=10^{\circ}$ plane to about 60 boundary-layer thicknesses in the $\theta=90^{\circ}$ plane. A comparison with the turbulent boundary-layer profiles (Fig. 6) shows the upstream propagation is greater in terms of actual distance for the laminar case, and in terms of the respective boundary-layer thicknesses the laminar propagation is 7 to 10 times that in the turbulent case. In the laminar case the propagation extended to the region of the most forward orifice, and the experimental data did not clearly indicate the maximum extent of the upstream propagation. The values quoted above are therefore conservative. The effect of the character of the boundary layer on the extent of the upstream pressure propagation is in qualitative agreement with experimental results for the interaction of shock waves and boundary layers on a flat surface. ${ }^{10,11}$

The pressure profiles show a plateau of slightly increased pressure, characteristic of the laminar case, extending upstream from the main shock pressure rise, indicating a bubble of dead air or region of flow separation. At the higher meridians the separation region is more extensive. The china-clay pattern in Fig. 4a indicates separation where the surface is still wet ahead of the Mach helix on the upper part of the body. The variation with $\theta$ of the extent of upstream propagation and separation is due in part to the cross flow of the low inertia air in the boundary layer, which results in an accumulation of air along the top of the body. This cross flow is produced by the component of the shockinduced velocity which is normal to the free-stream direction. Figs. $4 \mathrm{a}$ and $4 \mathrm{~b}$ show that the cross flow is stronger in the laminar boundary layer and illustrate the deviation of the flow near the body far ahead of the Mach helix, which is characteristic of the laminar case.

The schlieren photograph, Fig. 3, shows considerable thickening of the laminar boundary layer ahead of point $b$. The plane portion of the shock wave originating at the wing leading edge is the black line a. The main pressure rise in the top meridional plane of the body commences at point $b$ and continues to point c. As the compressive Mach waves travel outward from the body, they coalesce into a shock wave evidenced by the dark line $d$. The light region e represents the Prandtl-Meyer expansion at the trailing edge of the wing. The flow overexpands slightly and is recompressed by the shock wave following the Prandt1-Meyer expansion.

The experimental pressure distributions for the laminar case in Figs. 6a, 6b, and 6d exhibit the initial overcompression that has been observed experimentally in investigations of the two-dimensional interaction of a shock wave and the laminar boundary layer on a flat surface. ${ }^{10,11}$ The degree of this overcompression appears to vary with the axial pressure gradient of the pressure jump. No overcompression is noted in the meridional plane $\theta=60^{\circ}$, Fig. 6c, where the gradient is relatively weak. The pressure rise associated with the 


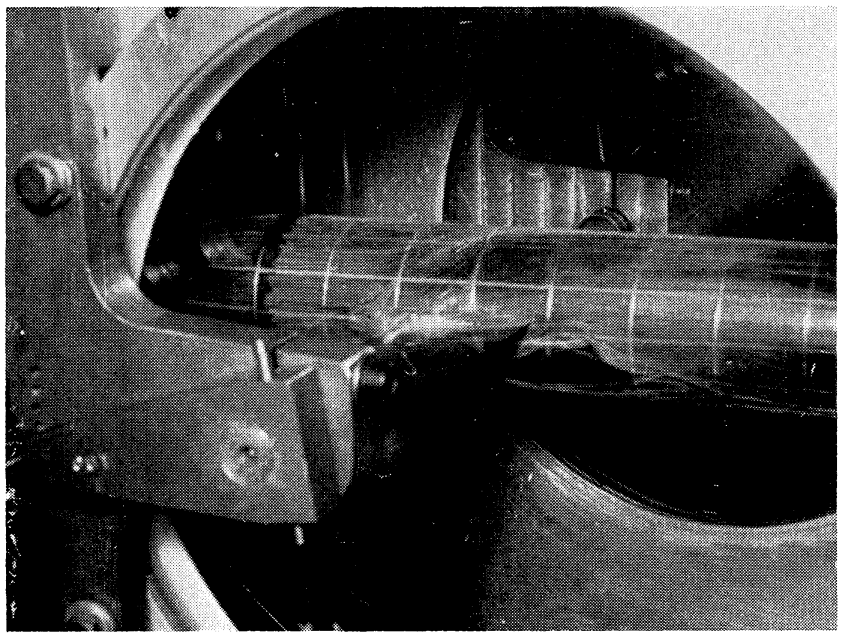

FIG. 1. Model in tunnel test section.

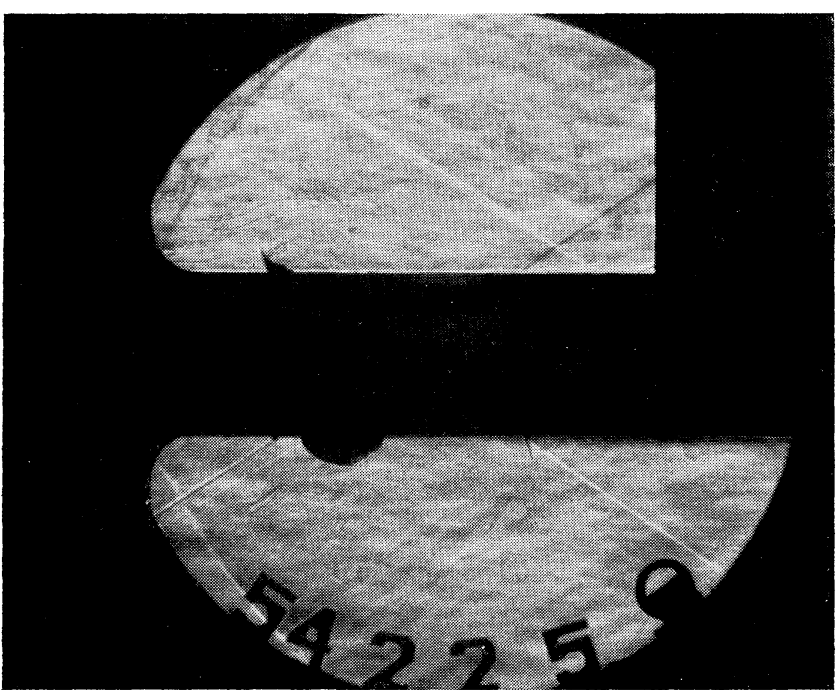

FIG. 2. Schlieren photograph, body alone.

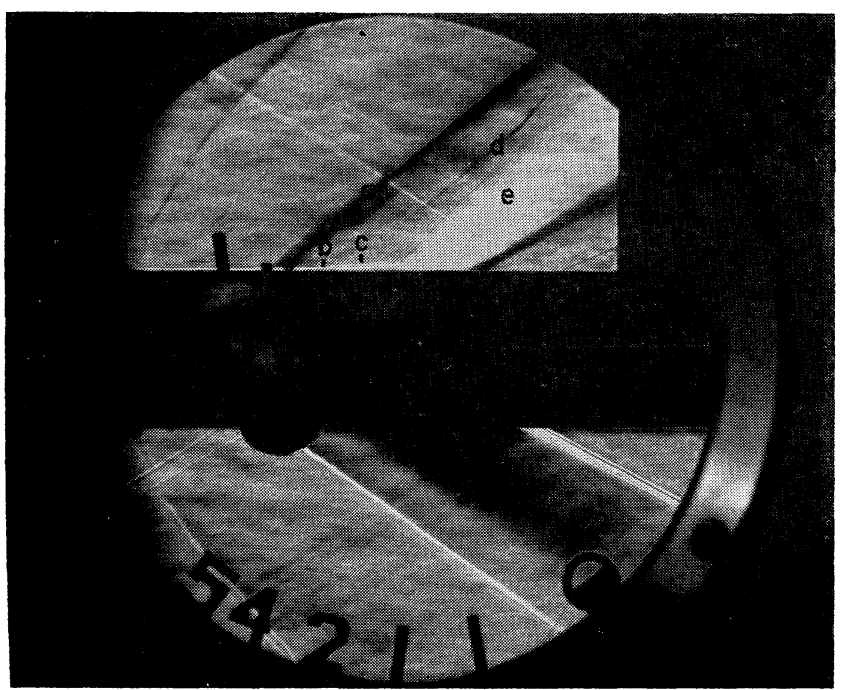

FIG. 3. Schlieren photograph, wing-body combination $\alpha_{W}=$ $-8.2^{\circ}$.

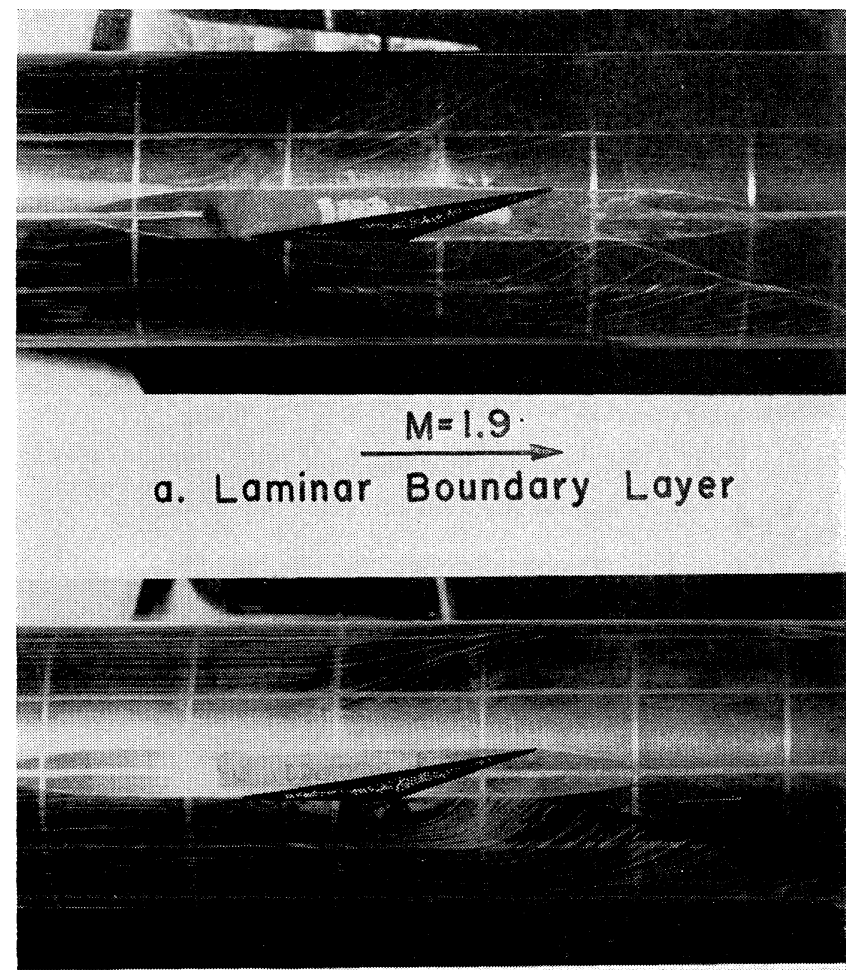

b. Turbulent $\underset{\text { Boundary Layer }}{\stackrel{M=1.9}{\longrightarrow}}$

FIG. 4. China-clay photograph, $\alpha_{B}=0, \alpha_{W}=-8.2^{\circ}$.
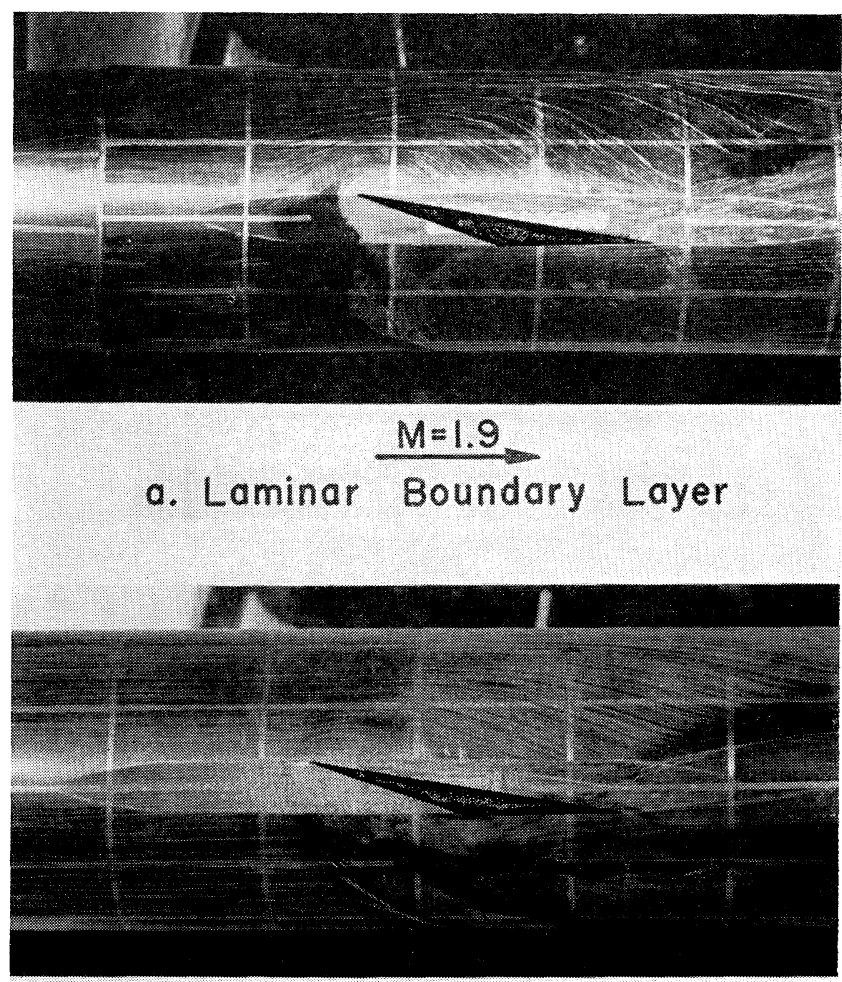

\section{$M=1.9$ \\ b. Turbulent Boundary Layer}

FIG. 5. China-clay photograph, $\alpha_{B}=0, \alpha_{W}=+8.2^{\circ}$ 


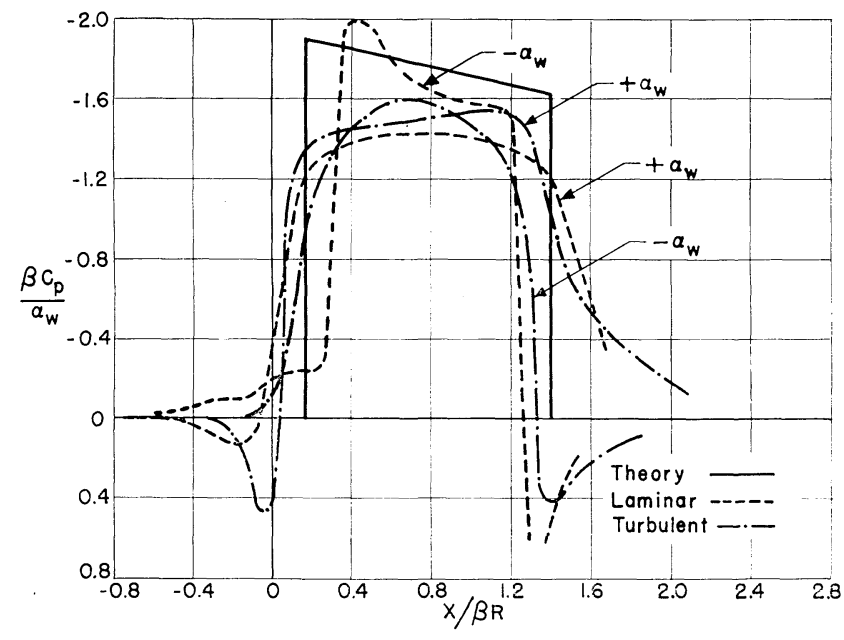

FIG. 6a. Body pressure profiles, $\theta=10^{\circ}$.

leading-edge shock occurs later for the laminar boundary layer than for the turbulent boundary layer. It is initiated at about the position of the actual trace of the leading-edge shock on the cylindrical body. Viscous effects result in a pronounced smoothing out of the pressure rise due to the shock.

The experimental maximum pressure coefficient for the laminar case agrees more closely with theory than that for the turbulent boundary layer.

At the trailing-edge expansion, for low values of $\theta$ both the laminar and turbulent pressure curves overshoot the ambient pressure and then begin to approach it asymptotically. The pressure behavior downstream of this point is uncertain due to flow nonuniformities and the reflection of the body nose shock which strikes in this region. The large disturbance from the wing-tip and wing support trunion also begin to affect the flow in this region.

\section{(2) Wing at $+8.2^{\circ}$ Angle of Attack}

For positive angles of attack an expansion wave originates at the leading edge of the wing. Experimentally, this expansion wave is preceded at small values of $\theta$ by a weak shock, probably a continuation of the strong shock wave on the compression side. It is a weaker compression in the laminar case but does not otherwise differ greatly from the turbulent phenomenon.

The upstream propagation of the pressure disturbance in the laminar case varies from about 20 boundary-layer thicknesses in the $\theta=10^{\circ}$ plane to about 30 boundary-layer thicknesses in the $\theta=90^{\circ}$ plane. This is again greater than the propagation observed with the boundary layer turbulent but somewhat less than for the negative wing angle of attack.

The expansion pressure profiles over the flat surface of the wing are similar for both the laminar and turbulent cases. However, the pressure rise is delayed slightly at the higher values of $\theta$ in the laminar case and the peak pressure coefficient is not as great. Due to the fan-like nature of the Prandtl-Meyer expansion, the axial pressure gradient is reduced as $\theta$ increases.

The pressure gradient on the body at constant $X / \beta R$ induces cross flow in the boundary layer, illustrated
TABLE 1

Interference Body Lift

Zero Length One Chord Length

Afterbody, 1b. Afterbody, $1 \mathrm{~b}$.

Linearized theory

Laminar boundary layer

Turbulent boundary layer

$\begin{array}{ll}0.612 & 2.993 \\ 0.644 & 3.014 \\ 0.991 & 3.263\end{array}$

by Figs. 5a and 5b. This cross flow is much more pronounced for the laminar boundary layer. The china-clay patterns again show the upstream flow deviation characteristic of laminar boundary-layer interaction, but no separation or pressure plateau is associated with an expansion.

A compression originates at the trailing edge, and the pressures begin to approach the ambient static pressure.

The maximum pressure coefficients over the flat surface of the wing at a positive angle of attack are considerably less than for the wing at negative angle of attack.

The above differences between the effects of interaction of an expansion wave and interaction of a shock wave with a laminar boundary layer have been experimentally observed elsewhere. ${ }^{5}$

\section{(3) Lift on the Body due to Wing-Body Interference}

The lift force on the body at zero angle of attack due to the presence of a flat-plate wing at an angle of attack was obtained by integrating the body pressure profiles. Since the lift is clearly dependent upon the amount of body downstream of the trailing edge, results were obtained for two different lengths of afterbody: (1) zero length-i.e., the body is assumed to terminate at the trailing edge of the wing; and (2) one chord length. The limitation of one chord length is necessary because of the effects of the disturbances mentioned in Section (C) (1) on the body downstream of this position. The results are presented in Table 1 as the lift force in pounds induced on the body by the wing.

The force on the body with laminar boundary layer slightly exceeds the force predicted by the linearized

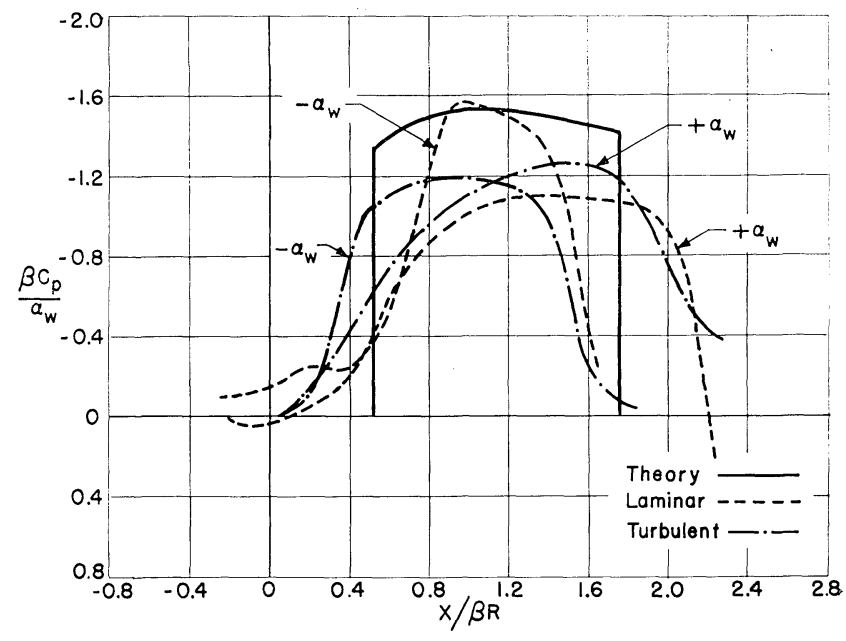

FIG. 6b. Body pressure profiles, $\theta=30^{\circ}$. 


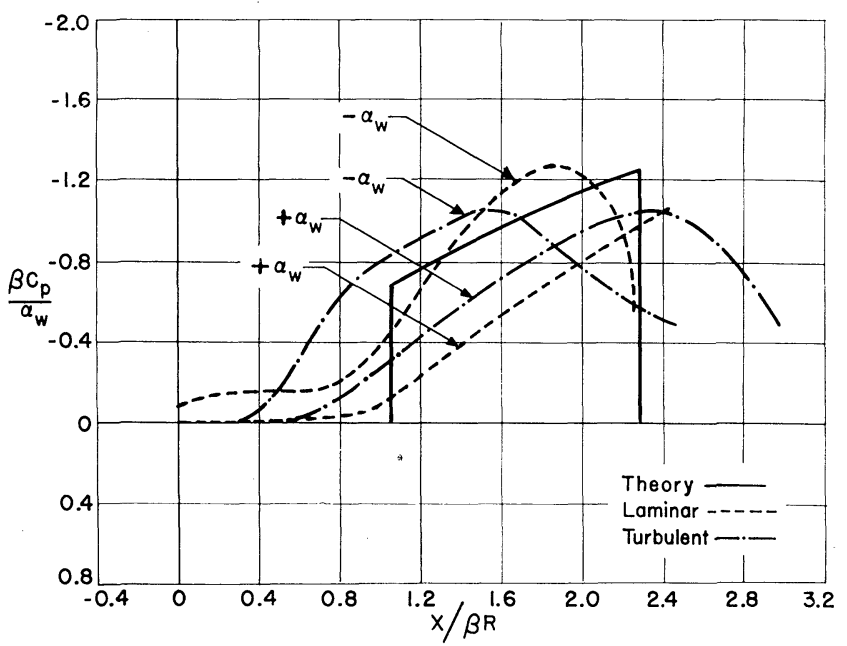

FIG. 6c. Body pressure profiles, $\theta=60^{\circ}$.

theory for both lengths of afterbody. In the turbulent case the experimental force exceeds the theoretical value by 62 per cent for zero afterbody and 9 per cent for one chord length afterbody.

The prediction of the interference lift on the body using the linearized theory appears conservative for the lengths of afterbody considered, regardless of the character of the boundary layer.

\section{(D) Conclusions}

With a laminar boundary layer on the fore body of the wing-body combination, the experimental pressure distribution on the body is in good agreement with linearized theory. With a turbulent boundary layer on the forebody the deviation is greater. The linearized inviscid theory ${ }^{3}$ appears adequate for the prediction of the body interference pressure distribution.

The singular viscous effects noted for the laminar boundary-layer interaction are: (1) upstream propagation of the pressure disturbance from the wing, as much as 60 boundary-layer thicknesses; (2) local separation of the laminar boundary layer, with a plateau of slightly increased pressure ahead of the shock pressure rise; (3) overcompression associated with sharp axial shock pressure gradients.

Although the viscous effects are strikingly evident in the pressure profiles for the laminar case, their effect on the body loading is not pronounced. For short lengths of afterbody the body loading in the turbulent case may differ substantially from the predicted value.

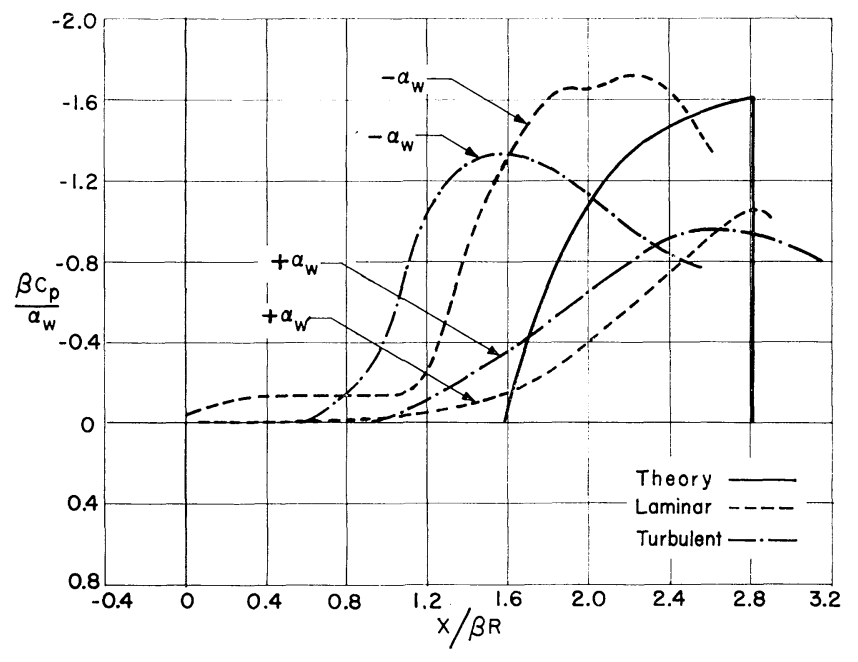

FIG. 6d. Body pressure profiles, $\theta=90^{\circ}$.

\section{REFERENCES}

${ }^{1}$ Lawrence, H. R., and Flax, A. H., Wing-Body Interference at Subsonic and Supersonic Speeds-Survey and New Developments, Journal of the Aeronautical Sciences, Vol. 21, No. 5, pp. 289-324, May, 1954.

2 Phinney, R. E., Wing-Body Interference; Progress Report No. 4, University of Michigan, Engineering Research Institute, 1952.

${ }^{3}$ Nielsen, J. N., and Pitts, W. C., Wing-Body Interference at Supersonic Speeds with an Application to Combinations with Rectangular Wings, NACA. TN 2677, April, 1952.

${ }^{4}$ Bailey, H. E., and Phinney, R. E., Final Report, Wing-Body Interference, Part I, Theoretical Invesiigation, University of Michigan, Engineering Research Institute, January, 1954.

${ }^{5}$ Pitts, William C., Nielsen, Jack N., and Gionfriddo, Maurice P., Comparison Between Theory and Experiment for Interference Pressure Field Between Wing and Body at Supersonic Speeds, NACA TN 3128, April, 1954.

${ }^{6}$ Bailey, H. E., and Phinney, R. E., Final Report, WingBudy Interference, Part II, Experimental Investigation of Cylindrical Model, University of Michigan, Engineering Research Institute, February, 1954.

${ }^{7}$ Bailey, H. E., and Phinney, R. E., Sume Experimental Results on Wing-Body Interference at Supersonic Speeds, Paper presented at the Second U.S. National Congress of Applied Mechanics, Ann Arbor, Mich., June 14-17, 1954.

${ }^{8}$ Garby, L. C., and Nelson, W. C., University of Michigan $8 \times 13$ Inch Intermittent-Flow Supersonic Wind Tunnel, University of Michigan, Memorandum 59, Engineering Research Institute, June, 1950

${ }^{9}$ Kuethe, A. M., and Schetzer, J. D., Foundations of Aerodynamics, p. 301; John Wiley \& Sons, Inc., 1950.

${ }^{10}$ Liepmann, H. W., Roshko, A., and Dhawan, S., On the Reflection of Shock Waves from Boundary Layers, NACA TN 2334, April, 1951.

${ }_{11}$ Barry, F. W., Shapiro, A. H., and Neumann, E. P., The Interaction of Shock Waves with Boundary Layers on a Flat Surface, Journal of the Aeronautical Sciences, Vol. 18, No. 4, pp 229-238, April, 1951. 